\title{
Assessing the Effect of Interaction Between C- Reactive Protein and Gut Microbiome on the Risks of Anxiety and Depression
}

\section{Yujing Chen}

Xi'an Jiaotong University

\section{Peilin Meng}

Xi'an Jiaotong University

\section{Shiqiang Cheng}

Xi'an Jiaotong University

\author{
Yumeng Jia \\ Xi'an Jiaotong University
}

\section{Yan Wen}

Xi'an Jiaotong University

\section{Xuena Yang}

Xi'an Jiaotong University

Yao Yao

Xi'an Jiaotong University

Chuyu Pan

Xi'an Jiaotong University

Chun'e Li

Xi'an Jiaotong University

Huijie Zhang

Xi'an Jiaotong University

Jingxi Zhang

Xi'an Jiaotong University

\section{Zhen Zhang}

Xi'an Jiaotong University

Feng Zhang ( $\square$ fzhxjtu@mail.xjtu.edu.cn )

Xi'an Jiaotong University

\section{Research}

Keywords: Gut microbiome, C-reactive protein (CRP), Depression, Anxiety 
Posted Date: April 27th, 2021

DOl: https://doi.org/10.21203/rs.3.rs-461660/v1

License: (c) (i) This work is licensed under a Creative Commons Attribution 4.0 International License. Read Full License 


\section{Abstract}

Cumulative evidence shows that gut microbiome can influence brain function and behavior via the inflammatory processes. However, the role of interaction between gut dysbiosis and C-reactive protein (CRP) in the development of anxiety and depression remains to be elucidated. In this study, a total of 3,321 independent SNP loci associated with gut microbiome were driven from genome-wide association study (GWAS). Using individual level genotype data from UK Biobank, we then calculated the polygenetic risk scoring (PRS) of 114 gut microbiome related traits. Moreover, regression analysis was conducted to evaluate the possible effect of interaction between gut microbiome and CRP on the risks of Patient Health Questionnaire-9 (PHQ-9) ( $N=113693)$ and Generalized Anxiety Disorder-7 (GAD-7) $(N=114219)$. At last, 11 candidate $\mathrm{CRP} \times$ gut microbiome interacting were detected for $\mathrm{PHQ}-9$ score, such as F_Ruminococcaceae $\left(\beta=-0.009, P=2.2 \times 10^{-3}\right), G \_$Akkermansia $\left(\beta=-0.008, P=7.60 \times 10^{-3}\right)$, F_Acidaminococcaceae $\left(\beta=0.008, P=1.22 \times 10^{-2}\right), G_{\text {_Holdemanella }}\left(\beta=-0.007, P=1.39 \times 10^{-2}\right)$ and O_Lactobacillales $\left(\beta=0.006 \otimes P=1.79 \times 10^{-2}\right) .16$ candidate $C R P \times$ gut microbiome interacting were detected for GAD-7 score, such as $O \_B a c t e r o i d a l e s ~\left(\beta=0.010, P=4.00 \times 10^{-4}\right)$, $O \_$Selenomonadales $(\beta=-0.010$, $\left.P=1.20 \times 10^{-3}\right), O \_$Clostridiales $\left(\beta=0.009, P=2.70 \times 10^{-3}\right)$ and $G_{-}$Holdemanella $\left(\beta=-0.008, P=4.20 \times 10^{-3}\right)$. Our results support the significant effect of interaction between CRP and gut microbiome on the risks of anxiety and depression, and identified several candidate gut microbiome for them.

\section{Introduction}

As common psychiatric disorders, the amount of people with depression and anxiety have increased over the past several decades, which leads to a growing concern in mental health research around the world(1). According to the report of WHO, the global population suffering from depression is estimated to be 322 million, while anxiety disorders affect more than 260 million people, accounting for $4.4 \%$ and $3.6 \%$ of the global population respectively, which resulted in a surge in suicide rates as well as a huge social and economic burden (2-4). However, there are elusive pathogenesis and lackluster treatments in anxiety and depression.

It is clear from previous studies that depression and anxiety are polygenic disorders, which are also influenced by the gut microbiome, systemic inflammation, and other environmental factors $(5,6)$. The gut microbiome of human contains diverse and dynamic populations of bacteria(7). Multiple human and animal studies suggested a strong association between the gut microbiome composition and the development of psychiatric disorders. For instance, it is demonstrated that host-associated microbial communities can affect basic developmental processes of the brain through the immune, metabolic or endocrine systems directly or indirectly (8). The finding for microbiota-gut-brain axis indicated a complex multiorgan bidirectional signaling system between the gut microbiome and the brain(9). Besides, gut microbiome could influence depression-like and anxiety-like behavior $(10,11)$. Individuals with depression could be identified from healthy subjects by single nucleotide exact amplicon sequence variants of gut 
microbiome(10). Nevertheless, the biological mechanism of gut microbiome affecting the development of psychiatric disorders remains largely unknown now.

As an acute-phase protein, CRP is associated with both pro-inflammatory and anti-inflammatory properties $(12,13)$. CRP plays a role in the recognition and clearance of foreign pathogens and damaged cells(14). It can activate the classic complement pathway and phagocytic cells(14). In addition, CRP affects psychiatric disorders through inflammatory processes. Parallel neural, humoral, and cellular interoceptive pathways can transmit inflammatory mediators to the brain to trigger alterations in mood and cognition motivation, and amplify behavioral stress responses(15). Inflammatory markers are wellknown etiological factors for psychiatric disorders, which could promote sickness behavior $(16,17)$. CRP was a key inflammatory marker for major depression(18). Increased peripheral blood CRP has been related to reduced functional connectivity between the left ventral striatum and ventromedial prefrontal cortex that correlated with the severity of anhedonia(19). People with symptoms of depression or anxiety frequently have increased level of CRP(20-22).

Gut microbiome could also affect inflammation status. Certain gut microbiome could lead to colitis with severe inflammation and overproduction of interleukin-17(23). In previous clinical trials, prebiotic could reduce the severity of hyperpnoea-induced bronchoconstriction and inflammatory markers such as CRP by regulating gut microbiome. These further indicated that gut microbiome is associated with inflammatory markers in chronic diseases (24). However, its exact mechanism remains unclear now. Further explorations are needed to draw a definitive conclusion.

In this study, data from UK biobank were applied to evaluate the influence of interactions between CRP and gut microbiome on anxiety and depression. Based on the significant SNPs associated with gut microbiota, PRS was firstly calculated. Linear regression was then conducted to evaluate the influence of CPR-gut microbiome interactions on the risks of anxiety and depression.

\section{Materials And Methods \\ 2.1. UK Biobank cohort}

Our study utilized the UK Biobank cohort (https://www.ukbiobank.ac.uk/), a prospective cohort study with a number of physical, health, and genetic data from approximately 500,000 individuals aged $40-69$. This large-scale biomedical database includes detailed lifestyle information as well as blood, urine, and saliva samples of participants. The UK Biobank genetic data contains genotypes of 488,377 participants. These were assayed using the UK BiLEVE Axiom array and UK Biobank Axiom array.

Imputation was carried out by IMPUTE4. Marker-based quality control was performed by using statistical tests. The metrics of missing rate and heterozygosity were computed to identify poor quality samples. Details of the array design, genotyping, and quality control procedures have been described previously (25). All data usage in this article is approved by UK Biobank (application 46478) and the Ethics Advisory Committee (EAC). 


\subsection{CRP measures in UK Biobank}

Our study contains 376,802 participants from UK Biobank with CRP data. The CRP was measured by immunoturbidimetric - high sensitivity analysis on a Beckman Coulter AU5800 when the participants were recruited and consent.

\subsection{Definition of depression and anxiety}

In this study, two common psychiatric disorders were analyzed, including depression and anxiety. Depression was measured based on PHQ-9 which is a classification algorithm used to screen for and measure depression severity(26). It focuses on nine depressive symptoms and signs, for example, Lack of interest or pleasure in doing things 20514, Recent feelings of depression 20510, Trouble falling or staying asleep, or sleeping too much 20517 , etc. The total score of it is $0-27$. Meanwhile, anxiety severity was measured by GAD-7 with a total score (0-21)(27). It focuses on seven anxious symptoms and signs, for example, Recent feelings or nervousness or anxiety 20506 , Recent inability to stop or control worrying 20509 , Recent worrying too much about different things 20520 , etc. The detailed definition was provided in the supplement. PHQ-9 score and GAD-7 were used as continuous variables in this study.

\subsection{GWAS data of gut microbiome}

The GWAS summary data sets of gut microbiome were derived from a recent large-scale study which included 114 gut microbiome related traits(28). Briefly, the 515F/806R primer pair was carried out to amplify the V4 region of the 16S rRNA gene for Flemish Gut Flora Project (FGFP) cohort individuals. Sequencing was carried out on the Illumina HiSeq platform. Fastq sequences were further analyzed per sample using the DADA2 pipeline (v.1.6). FGFP genotype data were phased using SHAPEIT3. Imputation was conducted by IMPUTE4 using UK10K and all 1000Genome Project phase 3 samples. After association analyses, 3,321 LD independent loci associated with 16S gut microbiome phenotypes were identified. Details of the array design, genotyping, and quality control procedures have been described previously(28).

\section{5. gut microbiome related PRS calculation and association analysis}

The gut microbiome related PRS of each subject was calculated by using individual SNP genotype data of the UK Biobank. Let PRSn denote the PRS value of gut microbiome for the nth subject, defined as:

$$
P R S_{n}=\sum_{i=1}^{l} E_{i} D_{i n}
$$

where / denotes the total number of gut microbiome analyzed in this study; $E_{i}$ denotes the effect size of significant gut microbiome associated SNP $i ; D_{i n}$ denotes the dosage of the risk allele of the ith SNP for the $n$th individual ( 0 is coded for homozygous protective genotype, 1 for heterozygous and 2 for 
homozygous polymorphic genotypes)(29). PLINK 2.0 was used to perform the PRS analysis. The linear regression model was established by R software (https://www.r-project.org/) to evaluate the possible associations among each gut microbiome PRS, CRP, and two psychiatric disorders. The PRSs of gut microbiome, CRP, and interaction of them were set as instrumental variables. PHQ-9 score and GAD-7 score are the outcomes adjusted by sex, age, Townsend Deprivation Index, and 10 principal components of population structure, respectively.

\section{Results}

\subsection{Interactions of gut microbiome and CRP for PHQ-9 score}

The basic characteristics of study samples were shown in Table 1 . We detected $11 \mathrm{CRP} \times \mathbf{x}$ gut microbiome interaction for PHQ-9 score,such as $F \_$Ruminococcaceae $\left(\beta=-0.009, P=2.2 \times 10^{-3}\right), G \_$Akkermansia $\left(\beta=-0.008, P=7.60 \times 10^{-3}\right), F \_$Acidaminococcaceae $\left(\beta=0.008, P=1.22 \times 10^{-2}\right), G \_$Holdemanella $(\beta=-0.007$, $\left.P=1.39 \times 10^{-2}\right)$ and $O \_$Lactobacillales $\left(\beta=0.006 \square P=1.79 \times 10^{-2}\right)$. The details were shown in Table 2 and Fig. 1.

\subsection{Interactions of gut microbiome and CRP for GAD-7 score}

We detected $16 \mathrm{CRP} \times$ gut microbiome interaction for anxiety GAD-7 score, like $O \_$Bacteroidales $(\beta=$ $\left.0.010, P=4.00 \times 10^{-4}\right)$, O_Selenomonadales $\left(\beta=-0.010, P=1.20 \times 10^{-3}\right), O \_$Clostridiales $(\beta=0.009, P=$ $\left.2.70 \times 10^{-3}\right)$ and $G_{-}$Holdemanella $\left(\beta=-0.008, P=4.20 \times 10^{-3}\right)$. The details were shown in Table 3 and Fig. 2.

\subsection{Common Interactions for both anxiety and depression}

We also compared the above association analysis results, found 4 common CRP $\times$ gut microbiome interactions for both PHQ-9 score and GAD-7 score: G_Holdemanella $\left(\beta=-0.007, P=1.43 \times 10^{-2}\right.$ for depression and $\beta=-0.008, P=4.30 \times 10^{-3}$ for anxiety), $G_{-}$Desulfovibrio $\left(\beta=0.007, P=2.64 \times 10^{-2}\right.$ for depression and $\beta=0.008, P=6.30 \times 10^{-3}$ for anxiety), $F_{-}$Coriobacteriaceae $\left(\beta=-0.006, P=4.57 \times 10^{-2}\right.$ for depression and $\beta=-0.005, P=4.46 \times 10^{-2}$ for anxiety) and $G \_$Barnesiella $\left(\beta=-0.006, P=3.16 \times 10^{-2}\right.$ for depression and $\beta=-0.006, P=4.96 \times 10^{-2}$ for anxiety)

\section{Discussion}

Although previous studies have found the functional relevance of gut microbiome and CRP with the development of anxiety and depression $(30,31)$, the biological mechanism underlying the effects of interaction between gut microbiome and CRP on the risks of anxiety and depression remains to be 
elucidated(32). In this study, we explored the interaction between CRP and 114 gut microbiome-related traits and observed a significant interaction between them for depression and anxiety.

Intestinal permeability defects are believed to be the basis for the chronic low-grade inflammation observed in stress-related psychiatric disorders(20). Inflammation takes an indirect role in modulating brain function. For example, gut microbiome could influence inflammation by beneficial nutrients (e.g. short-chain fatty acids (SCFAs)). It is clear that SCFAs could promote the expression of anti-inflammatory IL-10 in macrophages and intestinal dendritic cells(33). Furthermore, complex polysaccharides can be converted into SCFAs by Ruminococcaceae which could degrade mucin, enhance systemic and antitumor immune responses $(34,35)$. Ruminococcaceae was associated with disorders of the central nervous system $(35,36)$. There were higher levels of Ruminococcaceae in APOE2/E3 genotype carriers, compared with APOE4 carriers, one of the strongest prevalent risk factors for neuropathology and Alzheimer's disease(36). It is clear that Ruminococcaceae distributed differently in bipolar disorder (BD) and Major depressive disorder (MDD)(37). In our study, Ruminococcaceae is the most significant taxon associated with PHQ-9 score. In addition, the gut microbiome serves as a barrier to enteropathogen infection. This barrier function may be disrupted by inflammatory substances(38). However, its exact mechanism remains unclear now. Further explorations are needed to draw a definitive conclusion.

In this study, we found 11 significant taxons associated with PHQ-9 score, such as Akkermansia, Lactobacillales and Coprococcus. Akkermansia muciniphila (Akk bacteria) can also degrade mucin, which is negatively related to inflammation and metabolic disorders $(39,40)$. It is demonstrated that genus Akkermansia and family Akkermansiaceae were consistently changed in both idiopathic rapid-eyemovement sleep behavior disorder and Parkinson's disease(41). In addition, microbial community profiling revealed reduction (e.g. Akkermansia, Lactobacillus) in the Adrenocorticotrophic hormone induced depression rat model(42). Anti-inflammatory properties have been displayed in several strains of Lactobacillus in vitro in human intestinal epithelial cells(43). Lactobacillus was implicated in gut-brain communication and had positive effects on stress and cognition(44). Coprococcus was related to the activity of the dopamine pathway, and also led to the production of butyrate (6). Loss of bacteria that produce the anti-inflammatory, barrier-strengthening molecule butyrate, could lead to a loss of protection against epithelial inflammation and gut barrier disruption(45). Furthermore, Coprococcus was associated with higher quality of life indicators and was also depleted in depression(46).

We also found 16 significant taxons associated with GAD-7 score. Bacteroidales is the most common microbial category in the human gut. It takes significant roles in metabolic pathways and immune system(47). Previous studies reported that acquired inter bacterial defense gene clusters in Bacteroidales species reside in the human gut microbiome. In a mouse model, taking oral human commensal Bacteroides fragilis corrected gut permeability, altered gut microbiome composition, and ameliorated defects in communicative, stereotypic, anxiety-like, and sensorimotor behaviors(48). Individuals with MDD showed enriched species for Bacteroides and depleted species for Blautia(47). Furthermore, Blautia can mediate beneficial anti-inflammatory effects(47). 
We observed 4 gut microbiome PRS interacting with CRP were associated with both PHQ-9 score and GAD-7 score in our study, which may be related to pathophysiology of anxiety and depression through the communication of peripheral inflammation to the brain. For example, 3-hydroxyoctadecaenoic acid (C18$3 \mathrm{OH}$ ) is an agonist of peroxisome proliferator activated receptor gamma. The production of it by bacteria could be one of the mechanisms implicated in the anti-inflammatory properties of probiotics. In addition, $\mathrm{C} 18-30 \mathrm{H}$ correlated with an increase in the abundance in Holdemanella(49). In a previous animal study, higher loading of Holdemanella and Desulfovermiculus were found in Obsessive-compulsive patients(50). The over-representation of Desulfovibrio is associated with gut mucosal injury and inflammatory pathology through releasing hydrogen sulfide(50). In addition, Desulfovibrio competes with butyrate-producing bacteria for the lactate which results in the production of higher amounts of propionic acid(51). This phenomenon led to autism-like manifestations in animals(51). Moreover, previous studies also observed higher abundance of Desulfovibrio in MDD(52).

To the best of our knowledge, this is a novel study to explore the relationship between psychiatric disorders and the interaction of gut microbiome and CRP. Our study is based on a large cohort study with a long follow-up as well as representative samples. However, several limitations should be pointed out. First, owing to all samples in this study are from European ancestry, the findings should be inferred to other races with caution. Second, the key elements that influence the accuracy of PRS for a specific trait are SNP heritability, genetic architecture, sample size of the discovery GWAS including insufficiently powered GWAS sample sizes for most complex traits, potential confounding in causal inference, and a lack of ancestral diversity. Due to the related loci relied on previous published GWAS, the results may be affected.

In summary, our results support the significant effect of interaction between CRP and gut microbiome on the risks of anxiety and depression, and identified several candidate gut microbiome for them. These findings may provide novel therapeutic targets for psychiatric disorders, and give insights into the mechanism of anxiety and depression. Further studies are eager to confirm our findings and clarify the more detailed mechanism of gut microbiome $\times$ CRP interaction in psychiatric disorders.

\section{Abbreviations}

GWAS: genome-wide association study; CRP: C-reactive protein; PRS: polygenetic risk scoring; PHQ: Patient Health Questionnaire-9; GAD: Generalized Anxiety Disorder-7; EAC: Ethics Advisory Committee; FGFP: Flemish Gut Flora Project; SCFAs: short-chain fatty acids; BD: bipolar disorder; MDD: Major depressive disorder; Akk bacteria: Akkermansia muciniphila; C18-30H: 3-hydroxyoctadecaenoic acid.

\section{Declarations}

\section{Acknowledgements}

We thank Jing Ye, Xiaomeng Chu, Chujun Liang, Bolun Cheng for up-front data collation. 


\section{Authors' contributions}

YC and FZ conceived and designed the study; YC and PM wrote the manuscript; All authors collected the data and SC carried out the statistical analyses; $\mathrm{CL}, \mathrm{CP}, \mathrm{HZ}, \mathrm{JZ}, \mathrm{ZZ}, \mathrm{YW}$ and $\mathrm{YJ}$ made preparations for the manuscript at first. All authors reviewed and approved the final manuscript.

\section{Funding}

This study was supported by the National Natural Scientific Foundation of China $(81673112,81703177)$, the Key projects of international cooperation among governments in scientific and technological innovation (2016YFE0119100), the Natural Science Basic Research Plan in Shaanxi Province of China (2017JZ024), and the Fundamental Research Funds for the Central Universities.

\section{Availability of data and materials}

The datasets used and/or analyzed during the current study are available from the corresponding author on reasonable request.

\section{Ethics approval and consent to participate}

Not applicable.

\section{Consent for publication}

Not applicable.

\section{Competing interests}

There's no conflict of interest.

\section{References}

1. Aaronson ST, Sears P, Ruvuna F, Bunker M, Conway CR, Dougherty DD, et al. A 5-Year Observational Study of Patients With Treatment-Resistant Depression Treated With Vagus Nerve Stimulation or Treatment as Usual: Comparison of Response, Remission, and Suicidality. Am J Psychiatry. 2017;174(7):640-8.

2. Hedegaard H, Curtin SC, Warner M. Suicide Rates in the United States Continue to Increase. NCHS Data Brief. 2018(309):1-8.

3. Wittchen HU, Jacobi F, Rehm J, Gustavsson A, Svensson M, Jonsson B, et al. The size and burden of mental disorders and other disorders of the brain in Europe 2010. Eur Neuropsychopharmacol. 2011;21(9):655-79. 
4. Friedrich MJ. Depression Is the Leading Cause of Disability Around the World. JAMA. 2017;317(15):1517.

5. Akula N, Barb J, Jiang X, Wendland JR, Choi KH, Sen SK, et al. RNA-sequencing of the brain transcriptome implicates dysregulation of neuroplasticity, circadian rhythms and GTPase binding in bipolar disorder. Mol Psychiatry. 2014;19(11):1179-85.

6. Beurel E, Toups M, Nemeroff CB. The Bidirectional Relationship of Depression and Inflammation: Double Trouble. Neuron. 2020;107(2):234-56.

7. Parker A, Fonseca S, Carding SR. Gut microbes and metabolites as modulators of blood-brain barrier integrity and brain health. Gut Microbes. 2020;11(2):135-57.

8. Sharon G, Sampson TR, Geschwind DH, Mazmanian SK. The Central Nervous System and the Gut Microbiome. Cell. 2016;167(4):915-32.

9. Cryan JF, O'Riordan KJ, Cowan CSM, Sandhu KV, Bastiaanssen TFS, Boehme M, et al. The Microbiota-Gut-Brain Axis. Physiol Rev. 2019;99(4):1877-2013.

10. Stevens BR, Roesch L, Thiago P, Russell JT, Pepine CJ, Holbert RC, et al. Depression phenotype identified by using single nucleotide exact amplicon sequence variants of the human gut microbiome. Mol Psychiatry. 2020.

11. Diaz Heijtz R, Wang S, Anuar F, Qian Y, Bjorkholm B, Samuelsson A, et al. Normal gut microbiota modulates brain development and behavior. Proc Natl Acad Sci U S A. 2011;108(7):3047-52.

12. Du Clos TW. Function of C-reactive protein. Ann Med. 2000;32(4):274-8.

13. Brouillet S, Boursier G, Anav M, De La Boissiere DB, Gala B, Ferrieres-Hoa A. A, et al. C-reactive protein and ART outcomes: a systematic review. Hum Reprod Update. 2020;26(5):753-73.

14. Nehring SM, Goyal A, Bansal P, Patel BC. C Reactive Protein. StatPearls. Treasure Island (FL)2021.

15. Savitz J, Harrison NA. Interoception and Inflammation in Psychiatric Disorders. Biol Psychiatry Cogn Neurosci Neuroimaging. 2018;3(6):514-24.

16. Na KS, Jung HY, Kim YK. The role of pro-inflammatory cytokines in the neuroinflammation and neurogenesis of schizophrenia. Prog Neuropsychopharmacol Biol Psychiatry. 2014;48:277-86.

17. Ihekweazu FD, Versalovic J. Development of the Pediatric Gut Microbiome: Impact on Health and Disease. Am J Med Sci. 2018;356(5):413-23.

18. Howren MB, Lamkin DM, Suls J. Associations of depression with C-reactive protein, IL-1, and IL-6: a meta-analysis. Psychosom Med. 2009;71(2):171-86.

19. Mehta ND, Stevens JS, Li Z, Gillespie CF, Fani N, Michopoulos V, et al. Inflammation, reward circuitry and symptoms of anhedonia and PTSD in trauma-exposed women. Soc Cogn Affect Neurosci. 2020;15(10):1046-55.

20. Appleton J. The Gut-Brain Axis: Influence of Microbiota on Mood and Mental Health. Integr Med (Encinitas). 2018;17(4):28-32.

21. Kohler CA, Freitas TH, Stubbs B, Maes M, Solmi M, Veronese N, et al. Peripheral Alterations in Cytokine and Chemokine Levels After Antidepressant Drug Treatment for Major Depressive Disorder: 
Systematic Review and Meta-Analysis. Mol Neurobiol. 2018;55(5):4195-206.

22. Jiang HY, Zhang $X, Y u ~ Z H$, Zhang Z, Deng M, Zhao JH, et al. Altered gut microbiota profile in patients with generalized anxiety disorder. J Psychiatr Res. 2018;104:130-6.

23. Ahmed I, Roy BC, Khan SA, Septer S, Umar S. Microbiome, Metabolome and Inflammatory Bowel Disease. Microorganisms. 2016;4(2).

24. Hollister EB, Riehle K, Luna RA, Weidler EM, Rubio-Gonzales M, Mistretta TA, et al. Structure and function of the healthy pre-adolescent pediatric gut microbiome. Microbiome. 2015;3:36.

25. Bycroft C, Freeman C, Petkova D, Band G, Elliott LT, Sharp K, et al. The UK Biobank resource with deep phenotyping and genomic data. Nature. 2018;562(7726):203-9.

26. Davis KAS, Cullen B, Adams M, Brailean A, Breen G, Coleman JRI, et al. Indicators of mental disorders in UK Biobank-A comparison of approaches. Int J Methods Psychiatr Res. 2019;28(3):e1796.

27. Kroenke K, Spitzer RL, Williams JB, Lowe B. The Patient Health Questionnaire Somatic, Anxiety, and Depressive Symptom Scales: a systematic review. Gen Hosp Psychiatry. 2010;32(4):345-59.

28. Hughes DA, Bacigalupe R, Wang J, Ruhlemann MC, Tito RY, Falony G, et al. Genome-wide associations of human gut microbiome variation and implications for causal inference analyses. Nat Microbiol. 2020;5(9):1079-87.

29. Dudbridge F. Polygenic Epidemiology. Genet Epidemiol. 2016;40(4):268-72.

30. Lizano P, Lutz O, Xu Y, Rubin LH, Paskowitz L, Lee AM, et al. Multivariate relationships between peripheral inflammatory marker subtypes and cognitive and brain structural measures in psychosis. Mol Psychiatry. 2020.

31. Yang Z, Li J, Gui X, Shi X, Bao Z, Han H, et al. Updated review of research on the gut microbiota and their relation to depression in animals and human beings. Mol Psychiatry. 2020;25(11):2759-72.

32. Cathomas F, Murrough JW, Nestler EJ, Han MH, Russo SJ. Neurobiology of Resilience: Interface Between Mind and Body. Biol Psychiatry. 2019;86(6):410-20.

33. Singh N, Gurav A, Sivaprakasam S, Brady E, Padia R, Shi H, et al. Activation of Gpr109a, receptor for niacin and the commensal metabolite butyrate, suppresses colonic inflammation and carcinogenesis. Immunity. 2014;40(1):128-39.

34. Dalile B, Van Oudenhove L, Vervliet B, Verbeke K. The role of short-chain fatty acids in microbiota-gutbrain communication. Nat Rev Gastroenterol Hepatol. 2019;16(8):461-78.

35. Gopalakrishnan V, Spencer CN, Nezi L, Reuben A, Andrews MC, Karpinets TV, et al. Gut microbiome modulates response to anti-PD-1 immunotherapy in melanoma patients. Science. 2018;359(6371):97-103.

36. D'Amato A, Di Cesare Mannelli L, Lucarini E, Man AL, Le Gall G, Branca JJV, et al. Faecal microbiota transplant from aged donor mice affects spatial learning and memory via modulating hippocampal synaptic plasticity- and neurotransmission-related proteins in young recipients. Microbiome. 2020;8(1):140. 
37. Zheng P, Yang J, Li Y, Wu J, Liang W, Yin B, et al. Gut Microbial Signatures Can Discriminate Unipolar from Bipolar Depression. Adv Sci (Weinh). 2020;7(7):1902862.

38. Shi N, Li N, Duan X, Niu H. Interaction between the gut microbiome and mucosal immune system. Mil Med Res. 2017;4:14.

39. Shin J, Noh JR, Chang DH, Kim YH, Kim MH, Lee ES, et al. Elucidation of Akkermansia muciniphila Probiotic Traits Driven by Mucin Depletion. Front Microbiol. 2019;10:1137.

40. Ottman N, Geerlings SY, Aalvink S, de Vos WM, Belzer C. Action and function of Akkermansia muciniphila in microbiome ecology, health and disease. Best Pract Res Clin Gastroenterol. 2017;31(6):637-42.

41. Nishiwaki H, Hamaguchi T, Ito M, Ishida T, Maeda T, Kashihara K, et al. Short-Chain Fatty AcidProducing Gut Microbiota Is Decreased in Parkinson's Disease but Not in Rapid-Eye-Movement Sleep Behavior Disorder. mSystems. 2020;5(6).

42. Song J, Ma W, Gu X, Zhao L, Jiang J, Xu Y, et al. Metabolomic signatures and microbial community profiling of depressive rat model induced by adrenocorticotrophic hormone. J Transl Med. 2019;17(1):224.

43. Romijn AR, Rucklidge JJ, Kuijer RG, Frampton C. A double-blind, randomized, placebo-controlled trial of Lactobacillus helveticus and Bifidobacterium longum for the symptoms of depression. Aust N Z J Psychiatry. 2017; 51(8):810-21.

44. Foster JA, McVey Neufeld KA. Gut-brain axis: how the microbiome influences anxiety and depression. Trends Neurosci. 2013;36(5):305-12.

45. Liu RT, Rowan-Nash AD, Sheehan AE, Walsh RFL, Sanzari CM, Korry BJ, et al. Reductions in antiinflammatory gut bacteria are associated with depression in a sample of young adults. Brain Behav Immun. 2020;88:308-24.

46. Valles-Colomer M, Falony G, Darzi Y, Tigchelaar EF, Wang J, Tito RY, et al. The neuroactive potential of the human gut microbiota in quality of life and depression. Nat Microbiol. 2019;4(4):623-32.

47. Yang J, Zheng P, Li Y, Wu J, Tan X, Zhou J, et al. Landscapes of bacterial and metabolic signatures and their interaction in major depressive disorders. Sci Adv. 2020;6(49).

48. Hsiao EY, McBride SW, Hsien S, Sharon G, Hyde ER, McCue T, et al. Microbiota modulate behavioral and physiological abnormalities associated with neurodevelopmental disorders. Cell. 2013;155(7):1451-63.

49. Pujo J, Petitfils C, Le Faouder P, Eeckhaut V, Payros G, Maurel S, et al. Bacteria-derived long chain fatty acid exhibits anti-inflammatory properties in colitis. Gut. 2020.

50. Scheepers IM, Cryan JF, Bastiaanssen TFS, Rea K, Clarke G, Jaspan HB, et al. Natural compulsive-like behaviour in the deer mouse (Peromyscus maniculatus bairdii) is associated with altered gut microbiota composition. Eur J Neurosci. 2020;51(6):1419-27.

51. El Aidy S, Ramsteijn AS, Dini-Andreote F, van Eijk R, Houwing DJ, Salles JF, et al. Serotonin Transporter Genotype Modulates the Gut Microbiota Composition in Young Rats, an Effect Augmented by Early Life Stress. Front Cell Neurosci. 2017;11:222. 
52. Simpson CA, Diaz-Arteche C, Eliby D, Schwartz OS, Simmons JG, Cowan CSM. The gut microbiota in anxiety and depression - A systematic review. Clin Psychol Rev. 2021;83:101943.

\section{Tables}

Table 1. Basic characteristics of study sample from UK Biobank

\begin{tabular}{|lccl|}
\hline & Sample & Age & Sex (Female) \\
\hline PHQ-9 $(\mathrm{M}=2.71, \mathrm{SD}=3.64)$ & 113693 & 56.23 & $63344(55.7 \%)$ \\
\hline $\mathrm{GAD}-7(\mathrm{M}=0.28, \mathrm{SD}=1.05)$ & 114219 & 56.22 & $63626(55.7 \%)$ \\
\hline Note. Age was described as Mean (standard deviation). & & \\
\hline $\begin{array}{l}\text { Abbreviation: } \mathrm{PHQ}, \text { Patient Health Questionnaire. GAD, general anxiety disorder. M, Mean. SD, } \\
\text { Standard Deviation }\end{array}$ & & \\
\hline
\end{tabular}

Table 2. Association between PHQ score and GUT microbiota $\times$ CRP

\begin{tabular}{|c|c|c|c|c|}
\hline \multirow[t]{3}{*}{ Outcome } & Instrumental & \multicolumn{3}{|c|}{ GUT microbiota $\times$ CRP } \\
\hline & \multicolumn{4}{|l|}{ GUT microbiota } \\
\hline & & Beta & $\mathrm{T}$ & P-value \\
\hline \multirow[t]{11}{*}{ PHQ-9 } & F_Ruminococcaceae & -0.009 & -3.07 & .0022 \\
\hline & G_Akkermansia & -0.008 & -2.67 & .0076 \\
\hline & F_Acidaminococcaceae & 0.008 & 2.51 & .0122 \\
\hline & G_Holdemanella & -0.007 & -2.46 & .0139 \\
\hline & O_Lactobacillales & 0.006 & 2.37 & .0179 \\
\hline & G_Coprococcus & -0.007 & -2.25 & .0246 \\
\hline & G_Desulfovibrio & 0.007 & 2.22 & .0263 \\
\hline & G_Barnesiella & -0.006 & -2.16 & .0309 \\
\hline & G_Acidaminococcus & 0.006 & 2.03 & .0422 \\
\hline & G_Coprobacter & 0.005 & 2.06 & .0394 \\
\hline & F_Coriobacteriaceae & -0.006 & -2.00 & .0455 \\
\hline
\end{tabular}

Table 3. Association between GAD score and GUT microbiota $\times$ CRP 


\begin{tabular}{|c|c|c|c|c|}
\hline \multirow[t]{3}{*}{ Outcome } & Instrumental & \multicolumn{3}{|c|}{ GUT microbiota $\times$ CRP } \\
\hline & \multicolumn{4}{|l|}{ GUT microbiota } \\
\hline & & Beta & $\mathrm{T}$ & P-value \\
\hline \multirow[t]{16}{*}{ GAD-7 } & O_Bacteroidales & 0.010 & 3.55 & .0004 \\
\hline & O_Selenomonadales & -0.010 & -3.23 & .0012 \\
\hline & O_Clostridiales & 0.009 & 3.00 & .0027 \\
\hline & G_Holdemanella & -0.008 & -2.86 & .0042 \\
\hline & G_Desulfovibrio & 0.008 & 2.73 & .0064 \\
\hline & G_Blautia & 0.008 & 2.69 & .0071 \\
\hline & K_Bacteria & 0.008 & 2.68 & .0074 \\
\hline & G_Dialister & -0.008 & -2.63 & .0085 \\
\hline & C_Clostridia & -0.008 & -2.57 & .0101 \\
\hline & G_Ruminococcus & -0.006 & -2.23 & .0255 \\
\hline & F_Streptococcaceae & 0.007 & 2.25 & .0248 \\
\hline & G_Sporobacter & -0.007 & -2.16 & .0307 \\
\hline & F_Porphyromonadaceae & 0.006 & 2.13 & .0330 \\
\hline & C_Deltaproteobacteria & -0.006 & -2.10 & .0354 \\
\hline & F_Coriobacteriaceae & -0.006 & -2.02 & .0436 \\
\hline & G_Barnesiella & -0.006 & -1.98 & .0478 \\
\hline
\end{tabular}

\section{Figures}


Fig.1. The GM interacting with CRP in depression

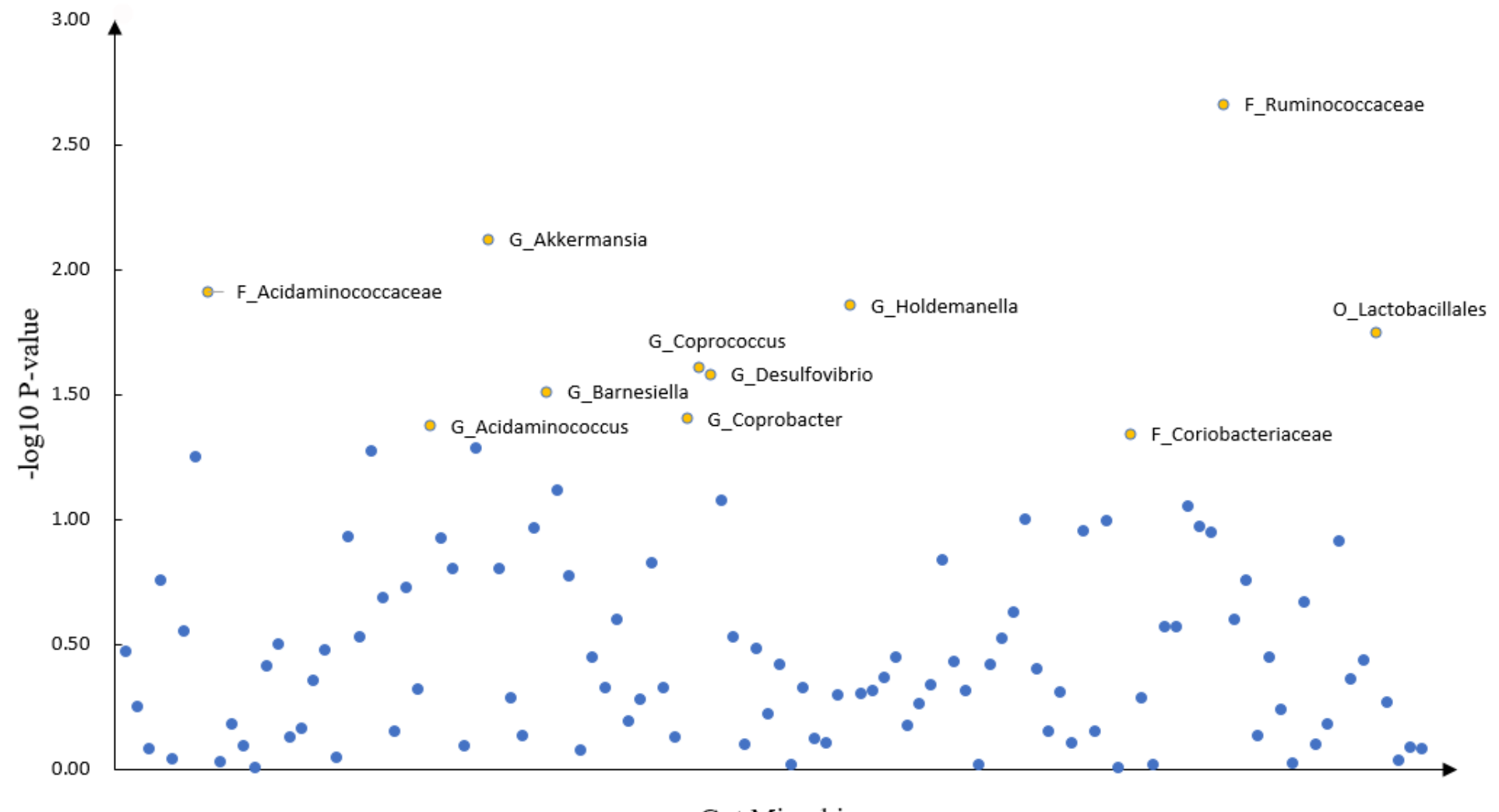

Gut Microbiome

\section{Figure 1}

The GM interacting with CRP in depression

Fig.2. The GM interacting with CRP in anxiety

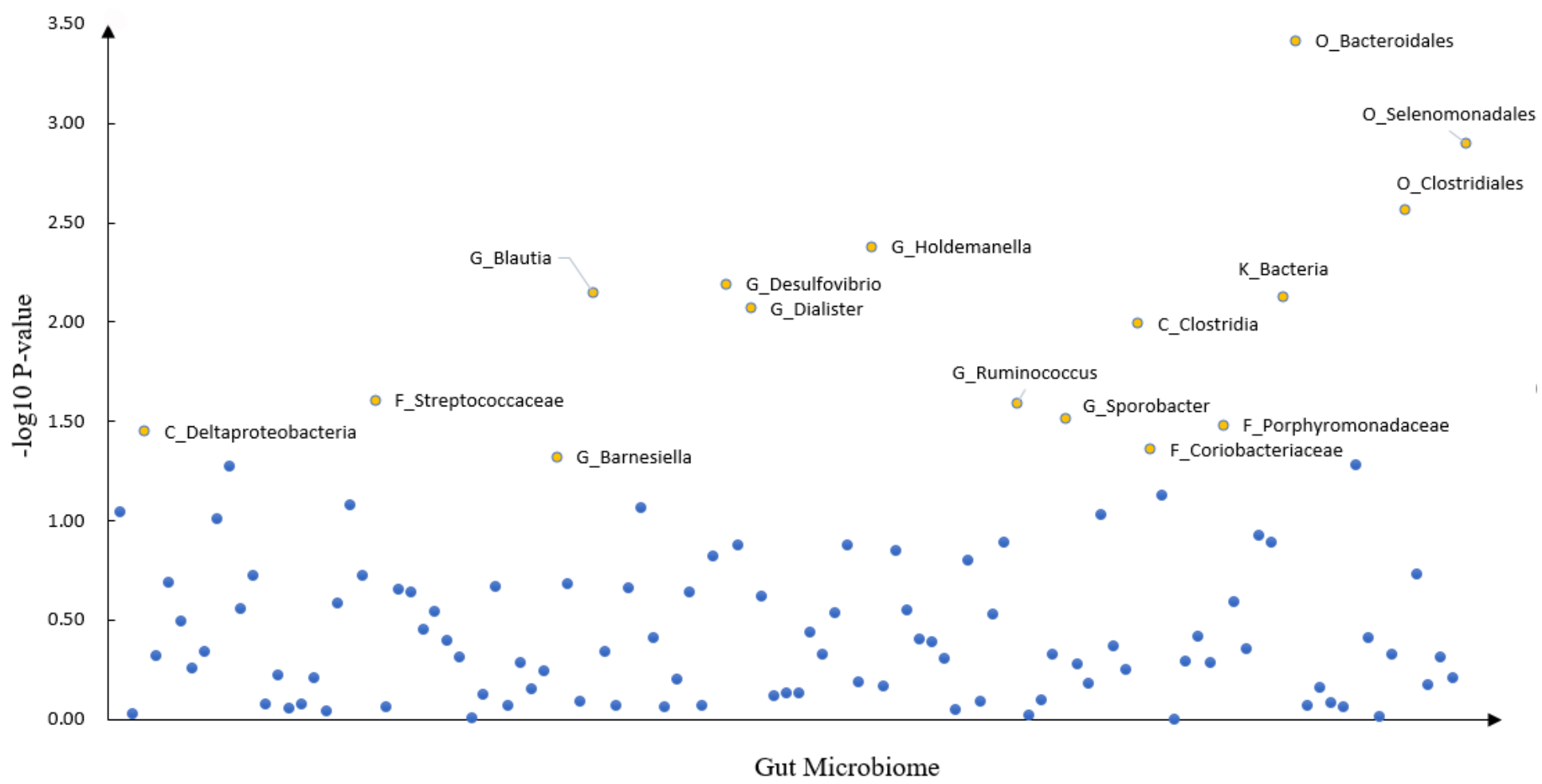


Figure 2

The GM interacting with CRP in anxiety

\section{Supplementary Files}

This is a list of supplementary files associated with this preprint. Click to download.

- Supplement.docx 